\title{
Redes, Ecología y Ciencias Sociales: las redes complejas en Ecología Humana
}

\author{
Miguel A. Munguía-Rosas ${ }^{\varpi}$; Salvador Montiel \& María T. Castillo \\ Centro de Investigación y de Estudios Avanzados del Instituto Politécnico Nacional (CINVESTAV), Unidad Mérida, Mérida, México.
}

\begin{abstract}
RESUMEN. Las redes complejas han probado ser una poderosa herramienta analítica en aspectos teóricos y prácticos en las Ciencias Sociales y la Ecología. A pesar de que la Ecología Humana derivó de estas disciplinas, las redes complejas en este campo del conocimiento están actualmente subutilizadas. Esto es preocupante porque las redes complejas podrían ayudar a entender y solucionar algunos desafíos socio-ambientales enfrentados por conservacionistas. Por ello, en esta revisión presentamos las bases teóricas generales y aplicaciones de las redes complejas en Ciencias Sociales y Ecología, así como novedosas y potenciales aplicaciones en Ecología Humana. El objetivo principal es despertar el interés de los académicos y tomadores de decisiones en el área socioambiental sobre las aplicaciones posibles de las redes complejas en este campo.
\end{abstract}

[Palabras clave: complejidad, ecosistemas, manejo de recursos naturales, sociedad]

\begin{abstract}
Networks, Ecology and Social Sciences: the complex network approach in Human Ecology: Complex networks have long been a powerful tool to assess theoretical and practical issues in Social Sciences and Ecology. Although Human Ecology emerged from these disciplines, the complex network approach has been underutilized in this field. This issue is particularly worrying because the complex network approach may help to understand and work out some socio-environmental challenges faced by conservationists. In this paper we revised the theoretical basis and applications of networks in Ecology and Social Sciences as well some novel and potential applications in Human Ecology. Our main goal is to increase awareness of academics and decision makers in the socio-environmental area regarding the applications of complex networks.
\end{abstract}

[Keywords: complexity, ecosystems, natural resources management, society]

\section{INTRODUCCIÓN}

La investigación y la aplicación de las redes complejas han tenido uno de sus mejores momentos en las últimas dos décadas debido al alto nivel de innovación. En el caso de las Ciencias Sociales y la Ecología, las redes complejas han tenido gran aceptación y sus aplicaciones se han diversificado de manera notable. Sin embargo, en Ecología Humana, a pesar de haberse derivado de las Ciencias Sociales y la Ecología, las aplicaciones de las redes complejas aún son limitadas. La Ecología Humana, entendida en este trabajo como el estudio de la relación entre los seres humanos y su medio ambiente (Marten 2001; Steiner 2002), es un campo novedoso con capacidad para contribuir en la solución de problemas socio-ambientales. Por lo tanto, en este artículo se exponen las bases conceptuales y, principalmente, las propiedades cuantitativas esenciales que describen las redes complejas. Con fines comparativos también se revisan algunas aplicaciones sobresalientes de estas redes en Ciencias Sociales y Ecología. Por último, se presentan y discuten algunas aplicaciones novedosas y potenciales en Ecología Humana. El objetivo principal es llamar la atención de académicos y tomadores de decisiones en el área socio-ambiental para que apliquen las redes complejas como herramienta metodológica en sus campos de acción.

\section{LAS REDES COMPLEJAS, ASPECTOS BÁSICOS}

Una red es una representación gráfica en dos dimensiones de un conjunto de elementos (nodos) y de las interacciones (enlaces) entre ellos. El adjetivo "complejo" alude a una de las propiedades más importantes de las redes: ser un conjunto de componentes (sistema) bajo una estructura variable cuyo comportamiento global no se puede predecir estudiando sus componentes en aislamiento. En un sistema complejo, sus componentes no están gobernados por reglas definidas, su sensibilidad es alta y tienen capacidad de autoorganización (Amaral \& Ottino 2007). Otra propiedad de las redes complejas es la de "mundo pequeño", que refiere a la existencia de atajos entre cualquier par de nodos, relativo a lo observado en una red 
con enlaces entre nodos dispuestos al azar (redes aleatorias). La propiedad de "mundo pequeño" es común en las redes del mundo real ya que es independiente del tamaño de la red. Otra propiedad que tienen las redes del mundo real es que -por lo general- algunos nodos están más conectados o tienen un mayor número de enlaces que otros (i.e., tienen mayor grado). En una red aleatoria, el grado tiene una distribución Poisson (i.e., muchos nodos con pocos enlaces), mientras que las redes del mundo real tienen una distribución con asimetría positiva más acentuada que lo esperado por una distribución Poisson. Como resultado de que existan sólo algunos nodos con muchos enlaces, la distribución del grado de las redes del mundo real tiene una distribución de cola muy larga a la derecha de la distribución. Esta distribución particular del grado que se le conoce como "distribución libre de escala" porque no hay una escala característica que las defina (Barabási \& Albert 1999). La distribución de cola larga del grado es causada porque los nuevos enlaces de una red son integrados preferencialmente a nodos preexistentes con mayor grado (Simon 1955). Aunque las redes complejas también ha sido utilizada para representar sólo de forma gráfica las relaciones entre nodos o procesos (Mc Guirre 2008), en este artículo se hace énfasis en la representación de las redes complejas donde, además del grafo, se presentan las propiedades que describen la red de forma numérica (Tabla 1).

Como ya indicamos en el párrafo anterior, las redes complejas se pueden clasificar en redes aleatorias y redes del mundo real, las primeras refieren a redes ficticias que tienen una conexión entre nodos por medio de enlaces arreglados al azar, mientras que las redes del mundo real describen algún sistema real donde, a diferencia de las redes aleatorias, los enlaces suelen formar atajos entre nodos. Otra clasificación de las redes se basa en los enlaces posibles entre tipos de nodo (Jordano et al. 2007). Por ejemplo, cuando los enlaces se pueden establecer entre cualquiera de los nodos de la red, se trata de redes unipartitas. A este tipo de redes pertenecen las redes sociales y las redes alimentarias. En contraparte están las redes bipartitas, donde sólo existen enlaces entre dos diferentes tipos de nodo. Un ejemplo claro de ellas son las interacciones planta-polinizador, donde los animales polinizadores representan un tipo de nodo y las plantas visitadas el otro tipo. Las redes unipartitas y bipartitas pueden clasificarse como redes dirigidas o redes no dirigidas, diferenciándose entre ellas sólo en términos de la dirección de la interacción entre nodos. La dirección de la interacción es útil cuando el flujo de la información tiene un sólo sentido (e.g., la relación emisor-receptor en redes de comunicación) o bien, cuando se tiene algún flujo direccional de energía (redes alimentarias). La Tabla 2 resume los tipos de clasificación de las redes complejas. Los lectores interesados en profundizar más sobre aspectos teóricos y metodológicos de las redes complejas pueden leer el trabajo de Amaral \& Ottino (2004).

\section{LAS REDES COMPLEJAS EN CieNCIAS SoCIALES Y ECOLOGÍA}

Tal vez el primer investigador social que hizo un análisis cuantitativo de una red social haya sido Jacob Moreno (1934), quien abordó la "huida" de 14 niñas en una escuela de Nueva York. Moreno analizó la relación social entre

Tabla 1. Descripción e interpretación de algunas de las propiedades más representativas de las redes complejas.

Table 1. Definition and meaning of some representative characteristics of complex networks.

\begin{tabular}{|c|c|}
\hline Propiedad & Descripción-interpretación \\
\hline Anidamiento & $\begin{array}{l}\text { El anidamiento mide la temperatura o grado de desorden de una matriz de interacción de la cual se } \\
\text { genera una red compleja bipartita. Alto anidamiento implica alta cohesión entre nodos con pocos } \\
\text { enlaces y nodos con muchos enlaces. }\end{array}$ \\
\hline Asimetría & $\begin{array}{l}\text { Se habla de asimetría cuando nodos con menor grado establecen enlaces de forma preferencial con } \\
\text { nodos con mayor grado. Las redes anidadas también son asimétricas, generalmente. }\end{array}$ \\
\hline Centralidad & $\begin{array}{l}\text { A nivel de red, mide que tan central es el nodo más central de la red relativo al resto de los nodos } \\
\text { de la red. La centralidad a nivel de nodo puede ser medida mediante el número de enlaces. Una red } \\
\text { con alta centralidad tiende a tener una forma de estrella. }\end{array}$ \\
\hline Densidad & $\begin{array}{l}\text { Promedio de enlaces por nodo. Se interpreta como una medida del nivel de interacción entre nodos } \\
\text { a nivel de toda la red. }\end{array}$ \\
\hline Grado & Número de enlaces por nodo. Describe el grado de conexión de un nodo focal con otros nodos de la red. \\
\hline Heterogeneidad & Número de nodos por cada tipo. Se interpreta como un indicador de la diversidad de actores. \\
\hline Integridad & Indicador de conexión de la red, integra las medidas de centralidad y densidad a nivel de red. \\
\hline Modularidad & $\begin{array}{l}\text { Se habla de estructura modular o modularidad cuando la red presenta subunidades o cliques. Cuando } \\
\text { las redes tienen una estructura modular existen grupos de nodos que interactúan entre ellos, pero } \\
\text { que no interactúan con nodos de otros grupos. }\end{array}$ \\
\hline
\end{tabular}


Tabla 2. Clasificación y criterios de clasificación de las redes complejas.

Table 2. Classes and classification criteria of complex networks.

\begin{tabular}{|c|c|c|}
\hline Criterio & Clasificación & Descripción \\
\hline \multirow[t]{2}{*}{ Sistema descrito } & $\begin{array}{l}\text { Redes del mundo } \\
\text { real }\end{array}$ & $\begin{array}{l}\text { Describen sistemas reales. El arreglo de los enlaces no es al } \\
\text { azar. La distribución del grado es libre de escala. }\end{array}$ \\
\hline & Redes aleatorias & $\begin{array}{l}\text { Describe sistemas ficticios. El arreglo de los enlaces es al azar. } \\
\text { La distribución del grado es aproximadamente Poisson. }\end{array}$ \\
\hline \multirow[t]{2}{*}{$\begin{array}{l}\text { Tipos y enlaces entre } \\
\text { nodos }\end{array}$} & Redes unipartitas & $\begin{array}{l}\text { Los enlaces pueden establecerse entre cualquier par de nodos } \\
\text { dentro de la red }\end{array}$ \\
\hline & Redes bipartitas & Los enlaces sólo pueden unir dos nodos de diferente tipo \\
\hline \multirow[t]{2}{*}{ Dirección del enlace } & Redes dirigidas & $\begin{array}{l}\text { La direccionalidad de la interacción se hace explicita en los } \\
\text { enlaces. }\end{array}$ \\
\hline & Redes no dirigidas & La direccionalidad de la interacción es desconocida. \\
\hline
\end{tabular}

las niñas y sugirió que la causa de dicha huida podría estar asociada con su posición en la red más que por razones imputables a cada niña. En los años cuarenta y cincuenta, las redes sociales se nutrieron de los avances en álgebra de matrices y teoría de grafos para formalizar su metodología y crear algunas propiedades cuantitativas ("metrics") (Luce \& Perry 1945). Tales avances, permitieron usar las redes sociales para poner a prueba hipótesis explícitas. Por ejemplo, Fisher (1948) puso a prueba el efecto de la urbanización en la cohesión social y para ello estudió 50 comunidades de California con diferente grado de urbanización. Sus resultados mostraron una relación negativa fuerte entre el grado de urbanismo y la densidad de la red (promedio de enlaces por nodo; Tabla 1). En la década del sesenta, los análisis de redes complejas influyeron en algunas corrientes de la Antropología Social. Las investigaciones en esta área de la Antropología se centraron en dos aspectos principales (revisado por Borgatti et al. 2009): combatir la visión de las sociedades humanas como entidades monolíticas e implantar la idea de que dichas sociedades constituyen una red de relaciones. Por otro lado, usar la estructura de la red como predictora de diversos aspectos sociales a nivel de individuo o pequeños grupos. Por ejemplo, Bott (1957) estudió la variación en la división de roles familiares de los cónyuges en familias británicas y encontró que la división de labores entre cónyuges es más evidente si la red familiar tiene una densidad elevada. En los años setenta, el interés fue más sociológico. Por ejemplo, Lorrain \& White (1971) encontraron que, mediante el colapso de nodos estructuralmente equivalentes (nodos con grado y posición similar en una red), era posible obtener una red reducida en la cual los nodos representaban posiciones estructurales más que individuos. En los años ochenta, las redes sociales se consolidaron como un programa de investigación, creándose la Organización Internacional de Redes Sociales, que edita la revista Social Networks y organiza un congreso anual sobre el tema. En la década del noventa y durante la primera década del siglo XXI, los resultados arrojados por el análisis de las redes sociales inspiraron aplicaciones en campos tan variados como la epidemiología (Cross \& Parker 2004), la criminología (Levy \& Pescosolido 2001) y la Ecología (ver siguiente párrafo).

Aunque las redes complejas tienen una aplicación más antigua en Ciencias Sociales que en Ecología, de ninguna manera se les puede considerar una aplicación nueva en esta última. A continuación revisaremos algunas aplicaciones de las redes complejas en los tres tipos principales de redes en Ecología: alimentarias, parasitoide-hospedante y de interacciones (Ings et al. 2009).

Las redes alimentarias son una abstracción de las relaciones alimentarias (enlaces) entre especies (nodos) dentro de una comunidad. Por lo general, las redes alimentarias son ricas en nodos y presentan niveles tróficos. El canibalismo es más frecuente en los niveles tróficos más altos. El generalismo alimentario (nodos con múltiples enlaces alimentarios) y la redundancia (nodos diferentes pero con interacciones alimenticias equivalentes) parecen ser constantes en este tipo de redes (Ings et al. 2009). Dentro de las redes alimentarias, las redes depredador-presa exhiben un patrón en el tamaño corporal de los nodos: los depredadores de talla mayor están en los niveles tróficos más altos y los más pequeños en los niveles más bajos (Otto et al. 2007). La energía en las redes complejas fluye generalmente de los individuos pequeños y abundantes, hacia los nodos en niveles tróficos más altos y menos abundantes (Reuman \& Cohen 2005) 
En la interacción parasitoide-hospedante, el parasitoide se desarrolla durante las etapas tempranas de su vida en un hospedante del cual se alimenta. Cuando el hospedante muere, el parasitoide inicia su etapa de vida libre (Wajnberg et al. 2008). Las redes parasitoidehospedante son bipartitas, ya que los nodos representan dos tipos de organismos sin enlaces entre sí. Los esfuerzos recientes en el estudio de las redes parasitoide-hospedante se han concentrado en estudiar su efecto indirecto sobre otros niveles tróficos (Lewis et al. 2002) y sobre la topología de la red (Bukovinszky 2008). También se han abordado los efectos que pueden tener las invasiones biológicas (Henneman \& Memmot 2001) y la degradación del hábitat (Tyianakis et al. 2007) sobre algunas propiedades de estas redes.

Las redes de interacciones son bipartitas. En el caso de las interacciones planta-animal, un grupo de nodos está representado por los animales y el otro por las plantas, los enlaces pueden representar visitas de los animales a las flores o consumo de frutos. Aunque en interacciones planta-planta resulta menos evidente la diferenciación de los dos grupos de nodos (porque ambos representan plantas), esto es posible en términos de la función que desempeñan en la interacción. Por ejemplo, en las redes de facilitación una planta es la proveedora de ciertos beneficios (e.g., sombra) mientras que otra planta es la beneficiaria. Las redes de interacción no representan sólo flujo de energía, sino la prestación de un servicio (polinización o dispersión de semillas). Las redes planta-polinizador, las redes plantafrugívoro y las redes planta-hormiga son las que han recibido mayor atención. La especialización de la interacción en redes mutualistas es relativamente mayor que en redes alimentarias (revisado por Ings et al. 2009). Se han encontrado importantes patrones en las redes de interacciones mutualistas; generalmente, estas redes son heterogéneas, anidadas y asimétricas (ver definiciones e interpretación de estas propiedades en Tabla 1; Bascompte \& Jordano 2007). Recientemente se ha estudiado la topología de redes bipartitas de facilitación entre plantas de zonas semiáridas (Verdú \& Valiente-Banuet 2008), y se encontraron similitudes con las redes mutualistas en términos de su propiedad más importante como el anidamiento. Este patrón de anidamiento también se ha encontrado en las redes epífita-forófito (Piazzon et al. 2011).

\section{Redes COMPLejas EN ECOlOGÍA HUMANA}

La Ecología Humana es un campo relativamente reciente que estudia la relación entre el ser humano y su medio ambiente (Marten 2001; Steiner 2002). Ya que el ser humano ha desarrollado una cultura compleja, estudiar su ecología necesariamente requiere del auxilio de las Ciencias Sociales. Si bien la aproximación de redes complejas ha sido de uso común en las Ciencias Sociales y en la Ecología por separado, no ha sido el caso para la Ecología Humana como campo integrador de ambas disciplinas. No obstante, en años recientes se han publicado algunos estudios novedosos que analizan las redes sociales en el contexto de manejo, gobernanza y contaminación de los recursos naturales, algunos de los cuales son analizados a continuación.

Por gobernanza ambiental se entiende el conjunto de procesos e interacciones entre actores sociales e instituciones respecto a la regulación delaprovechamientodelos recursos naturales (Mertens et al. 2011). Esta definición lleva implícita la idea de que existe una red de elementos cuyo interés común son los recursos naturales, por lo tanto, la estructura de esta red también puede tener repercusión en ciertos aspectos de la gobernanza de estos recursos. Bodin \& Crona (2009) revisaron recientemente los escasos trabajos cuantitativos sobre redes y gobernanza, y trataron de identificar qué aspectos estructurales de las redes pueden estar correlacionados con la gobernanza. Aunque estos autores detectaron que la densidad de la red puede favorecer una mejor gobernanza de los recursos naturales (vía fortalecimiento de la acción colectiva y la difusión de innovaciones del conocimiento), a densidades muy altas la gobernanza se ve debilitada por la homogeneización del conocimiento. Otras propiedades de las redes sociales que podrían estar asociados a diversos aspectos de la gobernanza fueron identificados por Bodin et al. (2006). De acuerdo con este estudio, ingredientes indispensables para un buen manejo o gobernanza de los recursos naturales como: la memoria social, la confianza y el aprendizaje, están íntimamente relacionados con la centralidad, la densidad y la modularidad (Tabla 1) de las redes respectivamente. $\mathrm{Al}$ igual que Bodin \& Crona (2009), estos autores identificaron que el incremento monotónico de cualquiera de estas 
propiedades puede traer efectos adversos para la gobernanza o el manejo.

Debido a la naturaleza dinámica del sistema humano- medio ambiente, es deseable que el manejo los recursos naturales sea adaptativo, es decir, que éste sea perfectible mediante un proceso de ensayo y error. Sandström \& Rova $(2010 \mathrm{a}, \mathrm{b})$ propusieron y pusieron a prueba la predicción de que la integración (Tabla 1) de los nodos (generalmente actores sociales o instituciones) y la heterogeneidad (Tabla 1) de las redes afectan positivamente la adaptabilidad de una experiencia de manejo. El razonamiento básico es que la integración incrementa la posibilidad de colaboración entre los nodos de la red para el establecimiento de las reglas de manejo, mientras que la heterogeneidad permite un acceso mayor a diferentes fuentes de conocimiento. La integración y la heterogeneidad son considerados aspectos críticos en el manejo adaptativo de recursos. Al poner a prueba la relación entre integración y heterogeneidad con adaptabilidad en estudios de caso, se encontró que, en general, se cumple la relación predicha. Sin embargo, parece existir un compromiso ("trade-off") entre la integración y la heterogeneidad por lo cual difícilmente se puede encontrar un nivel óptimo de adaptabilidad en el manejo de recursos. Estos autores señalan que son necesarios nuevos estudios para probar de forma robusta el efecto de diversas combinaciones de integración y heterogeneidad sobre la adaptabilidad del manejo y gobernanza de los recursos naturales (Sandström \& Rova 2010a,b).

Además de la estructura unipartita típica de las redes sociales (Tabla 2 ), más recientemente se han identificado otras variantes en manejo y gobernanza de recursos naturales, por ejemplo, las redes bipartitas en las que se pueden identificar diferentes tipos de actores. Se han usado redes bipartitas (Tabla 2) para identificar instituciones puente (nodos históricamente separados y distintos en términos de recursos y personas en relación a la red que tratan de ligar) e investigar su importancia en el manejo y gobernanza de recursos. Rathwell \& Peterson (2012) estudiaron los vínculos en cuanto al manejo del agua entre 34 municipios de Canadá, y encontraron que los municipios están conectados por organizaciones no gubernamentales (55 enlaces), mientras que sólo dos municipios tienen una relación directa. Las organizaciones no gubernamentales actúan como instituciones puente entre municipios, por lo que este resultado demuestra la importancia que tienen las instituciones puente en la vinculación de actores en el marco de manejo de recursos naturales, así como del potencial de las redes bipartitas para identificar el papel que juegan distintos tipos de nodos en una red. Otra variante estructural de una red social unipartita, en el contexto de manejo de recursos, es el tipo núcleo-periferia (Tabla 2). Este tipo de redes se caracterizan por tener dos elementos distintivos: un núcleo bien conectado y nodos periféricos pobremente vinculados entre ellos pero moderadamente conectados con el núcleo. Ernstson et al. (2008) estudiaron el caso de un parque urbano en Suecia donde intervenían 62 organizaciones civiles, la red tiene una estructura núcleoperiferia donde organizaciones altamente conectadas y dedicadas a la conservación están en el núcleo de la red, mientras que organizaciones civiles con mayor número de usuarios del recurso se mantienen en la periferia. De acuerdo con los autores, esta estructura es altamente funcional dado el tamaño de la red y su reciente formación. Con esta estructura se tiene un grupo central que coordina las acciones de conservación, mientras que la periferia actúa como sensor de diversos sucesos que comunican al núcleo de la red.

Las redes sociales asociadas al manejo y gobernanza de recursos naturales pueden tener una estructura contrastante, aunque se mantenga fijo el número y la identidad de los nodos, según qué aspecto está siendo representado por los enlaces. Lauber et al. (2008) estudiaron el manejo colaborativo de recursos naturales en algunas localidades de los Estados Unidos. Estos autores analizaron tres tipos de redes: de ideas, de influencia y de financiamiento. Aunque los nodos son estructuralmente los mismos, la densidad es mayor y bidireccional en las redes de las ideas, mientras que en redes de financiamiento, éstas son menos densas y las relaciones son unidireccionales.

Al igual que en otros campos de interés para los ecólogos humanos, el estudio de la contaminación ambiental y los riesgos que ésta representa para la salud humana, pocas veces se ha llevado a cabo con una aproximación cuantitativa de redes complejas. Sin embargo, existe un caso bien documentado sobre el papel que juega la estructura de las redes sociales en la concienciación de la contaminación ambiental y en el cambio de conductas para 
reducir sus efectos adversos sobre la salud humana (Mertens et al. 2008; 2011; 2012). Esta serie de estudios quizás representan el único caso bien documentado del impacto al mediano plazo de la educación participativa sobre la estructura de redes de discusión acerca de la contaminación y sus repercusiones en la salud. Por considerarlo ilustrativo, esta sección resume las aproximaciones y resultados generados por este programa de investigación.

El proyecto CARUSO ha usado el enfoque de redes complejas (entre otros) para analizar los resultados de una investigación participativa sobre la contaminación por mercurio en la Amazonia brasileña, región en la que algunas prácticas agrícolas (e.g., roza-tumba y quema) han generado un incremento en la erosión del suelo y el consecuente transporte del mercurio hacia los cuerpos de agua. El mercurio ingresa a los humanos por medio del consumo de peces y se ha identificado que los peces con mayor contenido de mercurio son los carnívoros (Mertens et al. 2012). Como parte del proyecto CARUSO se han desarrollado talleres participativos sobre el problema que representa el mercurio para el ecosistema y la salud humana, usando como herramienta analítica las redes complejas para entender cómo la estructura de la red se relaciona con conductas y la adopción de nuevos hábitos. Algunos resultados revelan que las redes de discusión sobre el mercurio presentan un mayor número de interacciones entre personas del mismo género y rara vez se dan entre personas de distinto género, lo que sugiere que una estrategia efectiva de concienciación sobre contaminación debe considerar ambos géneros, de otra manera, se corre el riesgo de que la información sólo se difunda entre una porción de la población (Mertens et al. 2005). También se ha detectado que el promedio de discusiones entre personas de la red es afectado por la edad; por ejemplo, jóvenes menores de 30 años discuten en menor medida sobre el tema. Este aspecto es preocupante ya que es la edad de mayor fertilidad y es posible la contaminación de los hijos durante el periodo de gestación (Mertens et al. 2005). La red de discusión sobre contaminación por mercurio de esta región es muy densa y relativamente robusta a perturbaciones. El colaborador del proyecto está involucrado con el 93\% de los enlaces con otros nodos, aunque existe el temor de que con la salida del colaborador la red de discusión sobre mercurio pierda densidad, el modelado de este evento sugiere que sólo se perdería $2 \%$ de los enlaces (Mertens et al. 2008).

Otro aspecto de importancia capital para el proyecto CARUSO ha sido impulsar un cambio en los hábitos alimentarios de los pobladores de la región para minimizar el impacto de la contaminación por mercurio en la salud humana. Por lo tanto, en los talleres participativos se enfatizó que el consumo de peces no carnívoros puede disminuir las probabilidades de intoxicación por mercurio. Mertens et al. (2012) elaboraron una red de difusión de una innovación, donde dicha innovación correspondía al conocimiento de que los peces carnívoros contenían mayor cantidad de mercurio que los otros. De acuerdo a los resultados, la gran mayoría de los pobladores están conectados por esta innovación, pero prefieren hablar del tema con personas de su mismo género, tal y como había advertido en un estudio previo (Mertens et al. 2005). De forma interesante, las mujeres con mayor escolaridad fueron las más propensas a adoptar la innovación alimenticia. Gracias al uso de redes complejas como herramienta analítica, este estudio fue capaz de evaluar el poder de la investigación participativa en la adopción de nuevas dietas y monitorear la difusión de un nuevo hábito alimentario.

\section{CONSIDERACIONES FINALES}

Como se puede constatar en este artículo, la variedad de aplicaciones y el número de estudios con redes complejas en Ciencias sociales y Ecología contrasta con lo encontrado en la Ecología Humana. Es interesante que, siendo la Ecología Humana un híbrido entre las Ciencias Sociales y la Ecología, el uso de las redes complejas sea limitado relativo a las disciplinas que la gestaron. Parte de la explicación radica en que la Ecología Humana es más reciente, pero para nosotros no es la razón principal. Consideramos que el aspecto más influyente es que el sujeto de estudio de la Ecología Humana es más complejo que sus componentes (sociedad humana y ecosistemas) por separado. Es decir, la interacción sociedad humana-ambiente tiene propiedades emergentes que no pueden ser abordadas desde el punto de vista puramente social o ecológico. Las propiedades emergentes de tal interacción puede ser abordadas y descritas de mejor manera con una aproximación de redes complejas (Amaral \& Ottino 2004), en comparación con otras aproximaciones enfocadas en el nivel de individuo o de 
población, sin considerar la interacción entre sus elementos a describir. Las aplicaciones de las redes complejas a la Ecología Humana descritas en este trabajo pueden considerarse como la forma más sencilla de abordar la complejidad desbordada por el sistema sociedad humana-medio ambiente: estudiando las redes sociales en condiciones o coyunturas ambientales relevantes para el ecólogo humano. Al ser la Ecología Humana el estudio de las relaciones entre el hombre y su ambiente, una red compleja en Ecología Humana también tendría que ser capaz de considerar nodos humanos y otros elementos del ecosistema (e.g., plantas y animales) dentro de la red. Sin embargo, no conocemos algún estudio que haya tomado esta aproximación (i.e., redes cuantitativas donde se identifiquen humanos y al mismo tiempo otros elementos del ambiente biótico o abiótico como nodos).

En Ciencias Sociales y Ecología se han usado las redes complejas desde hace décadas para la prueba de hipótesis. En contraste, los pocos estudios disponibles sobre redes complejas en Ecología Humana son principalmente estudios de caso. Fue hasta no hace mucho que se publicaron los primeros estudios comparativos (Sandström \& Rova 2010b) y las revisiones (Bobin \& Crona 2009), donde se ha puesto a prueba algunas predicciones o se analizaron los patrones en la estructura de las redes en Ecología Humana. Aunque todos los intentos de búsqueda de patrones o de estudios comparativos señalan que los estudios son pocos aún, los patrones preliminares se pueden usar para formular predicciones en estudios futuros. Las relación entre integridad y heterogeneidad como predictoras de la adaptabilidad del manejo de recursos naturales y la gobernanza (Sandströn \& Rova 2010a,b), es un claro ejemplo de un patrón preliminar que requiere se ponga a prueba en otros contextos. También, es sumamente interesante que estudios independientes (Ernston et al. 2008; Sandström \& Rova 2010a) hayan detectado la existencia de compromisos entre diferentes parámetros estructurales de las redes, así como la falta de relación lineal entre parámetros de la red (e.g., densidad) y algunas características deseables de manejo o gobernanza de los recursos naturales (e.g., adaptabilidad) (Bodin \& Crona 2009). Estas observaciones sugieren que: i) valores altos de cualquier parámetro dela red estarán asociados a valores bajos en otros y, por lo tanto, valores inusualmente altos en un parámetro de la red son indicadores de desequilibrio. Por otro lado ii), los parámetros de las redes presentan un punto óptimo (en términos del efecto asociado que tienen sobre el manejo o la gobernanza de recursos naturales). En otras palabras, los valores extremos en la mayoría de los casos generan resultados subóptimos.

El uso de las redes complejas en Ecología Humana, como en otras áreas, han servido para identificar patrones, mismos que parecen ser comunes en gran variedad de sistemas (e.g., telecomunicaciones, ecosistemas, sociedades). Esta convergencia es lo que ha sorprendido y sigue motivando la aplicación de las redes complejas en diferentes sistemas. Un reto común en todas las áreas donde se ha usado las redes complejas para describir patrones, es encontrar las causas subyacentes de esos patrones, tarea que, en el caso de la Ecología Humana, podría ser apoyada con otras aproximaciones a profundidad (e.g., etnografía). El objetivo de este trabajo no es que el ecólogo humano sustituya las herramientas que tradicionalmente ha utilizado por las redes complejas, sino que las incorpore dentro de su repertorio metodológico.

Resulta notable que la mayoría de los estudios de redes en el campo de la Ecología Humana se han realizado en países europeos y de América del Norte, por lo que es necesario realizar estudios en países en vías de desarrollo para tener un panorama geográfico y social más amplio y pertinente para nuestra sociedad latinoamericana. Sabemos que en esta región la diversidad cultural y biológica son altas y están íntimamente relacionadas (Boege 2008), por lo que no es difícil predecir que estudiar con un enfoque de redes complejas aspectos de Ecología Humana en Latinoamerica será más desafiante. El interés creciente que se ha dado en los últimos años a la dimensión social de la conservación de los recursos naturales podría verse fortalecido si se abordara desde una perspectiva de redes complejas, dadas las virtudes de las redes complejas para: i) identificar actores clave en un grupo, ii) estudiar el sistema como un todo y iii) modelar (y de esta manera predecir) los efectos de la integración y/o la pérdida de nodos sobre la topología de la red. También es preocupante que pese a la relevancia de entender la interacción hombre-medio ambiente, el número de instituciones y de investigadores dedicados a esta área del conocimiento sea muy limitada. Por ejemplo, en México sólo existe una institución que ofrece un programa de posgrado en Ecología Humana y ninguna 
universidad de este país ofrece formación en Ecología Humana a nivel de Licenciatura. En conclusión, las redes complejas pueden complementar las herramientas disponibles actualmente $\mathrm{y}$, en conjunto, dar cuenta de diversos aspectos críticos de la relación entre la sociedad humana y su ecosistema.

\section{BIBLIOGRAFÍA}

Amaral, LAN \& JM Ottino. 2007. Augmenting the framework for the study of complex systems. Eur J Phys, 38:147-162.

BÁRBASI, AL \& R AlBERT. 1999. Emergence of scaling in random networks. Science, 286:509-512.

BASCOMPTE, J \& P JoRDANO. 2007. Plant-animal mutualistic networks: The architecture of biodiversity. Annu Rev of Ecol Evol Syst, 38:567-93.

Bodin, O; B CRONA \& H ERNSTON. 2006. Social networks in natural resources management: what is there to learn from a structural perspective? Ecol Soc, 11:2.

Bodin, O \& B CRONA. 2009. The role of social networks in natural resource governance: What relational patterns make a difference? Glob Environ Change, 19:366-374.

BOEGE, E. 2008. El patrimonio biocultural de los pueblos indígenas de México. INAH-CDI. Distrito Federa, México. Pp. 342.

Borgatti, SP; A Mehra; DJ Brass \& G Labianca. 2009. Network analysis in social sciences. Science, 323:892-895.

Bотт, E. 1957. Family and social network. Tavistok. Londres, UK. Pp. 320.

BUKOVINSZKY, T; FJF VAN-VeEN; Y JONGEMA \& M DiCKe. 2008 Direct and indirect effects of resource quality on food web structure. Science, 319:804-807.

CROSS, R \& A PARKER. 2004. The hidden power of social networks. Harvard Business School. Boston, EU. Pp. 304.

ERnstson, H; S Sörlin \& T ElmQvist. 2008. Social movements and ecosystem services-the role of social network structure in protecting and managing urban green areas in Stockholm. Ecol Soc, 13:39.

FISCHER, CS. 1948. To dwell among friends: personal network in town and city. University of Chicago Press. Chicago, EU. Pp. 459.

HeNNEMAn, ML \& J Meмmotт. 2001. Infiltration of hawaiian community by introduced biological control agents. Science, 293:1314-1316.

InGS, C; JM MONTOYA; J BASCOMPTE; N BLÜTGen; L BRown; ET AL. 2009. Ecological networks: beyond food webs. J Anim Ecol, 78:253-269.

Jordano, P; D VÁzquez \& J Bascompte. 2007. Redes complejas de interacciones mutualistas planta-animal, Capítulo 1. Pp. 17-41. En: Medel, R; M Aizen \& R Zamora (eds.). Ecología y evolución de las interacciones plantaanimal: conceptos y aplicaciones.Editorial Universitaria. Santiago de Chile. Pp. 400.

LAUBER, TB; DJ DeCKER \& BA KNUTH. 2008. Social networks and community-based natural resource management. Environ Manage, 42:677-687.

LeVY, JA \& BA Pescosolido. 2002. Social networks and health. Elsevier. Londres, UK. Pp. 380.

Lewis, OT; J Memmott; J Lasalle; CHC Lyal, C Whitefoord; ET AL. 2002. Structure of a diverse tropical forest insectparasitoid community. J. Anim Ecol, 71:855-873.
LORRAIN, FP \& HC WHITE. 1971. Structural equivalence of individuals in social networks. J Math Sociol,1:49-80.

LuCE, R \& A PERry.1945. A method of matrix analysis of group structure. Psichometrika, 14:95-116.

McGuirRe, SJ. 2008. Securing access to seeds: social relations and sorghum seed exchange in eastern Ethiopia. Hum Ecol, 36:217-229.

Marten, GG. 2001. Human ecology. Routledge. Londres, UK. Pp. 256.

Mertens, F; J Saint-Charles; D Mergler, CJ Passos \& M LuCOTTE. 2005. Network approach for analyzing and promoting equity in participatory ecohealth research. Ecohealth, 2:113-126.

Mertens, F; J Saint-Charles; M Lucotte \& D Mergler. 2008. Emergence and robustness of a community discussion network on mercury contamination and health in the Brazilian Amazon. Health Educ Behav, 35:509-521.

Mertens, F; R Távora; IF da-Fonseca, RL Grando, M CAstro; et AL. 2011. Redes sociais, capital social e governança ambiental no Território Portal da Amazônia. Acta Amazon, 41:481-492.

Mertens, F; J Saint-Charles \& D Mergler. 2012. Social communication network analysis of the role of participatory research in the adoption of new fish consumption behaviors. Soc Sci Med, 75:643-650.

MORENO, JL. 1934. Who shall survive? Nervous and mental disease publishing company. Washington DC, EU. Pp. 763.

Otto, SB; BC Rall \& U Brose. 2007. Allometric degree distributions facilitate food-web stability. Nature, $\mathbf{4 5 0}$ : 1226-1203.

Piazzon, M; AR Larrinaga \& L Santamaría. 2011. Are nested networks more robust to disturbance? A test using epiphyte-tree, comensalistic networks. PloS One, 6:e19637.

Rathwell, KJ \& GD Peterson. 2012. Connecting social networks with ecosystem services for watershed governance: a social-ecological network perspective highlights the critical role of bridging organizations. Ecol Soc,17:24.

Reuman, DC \& JE CoHEN. 2005. Estimating relative energy fluxes using the food web, species abundance, and body size. Adv Ecol Res, 36:137-182.

SANDSTRÖM, A \& C Rova. 2010a. The network structure of adaptive governance: a single case study of a fish management area. International Journal of the Commons, 4:528-551.

SANDSTRÖM, A \& C ROVA. 2010b. Adaptive co-management networks: a comparative analysis of two fishery conservation areas in Sweden. Ecol Soc, 15:14.

SIMON, HA. 1955. On a class of skew distribution functions. Biometrika. 42:425-440.

SteINER, DF. 2002. Human Ecology: following nature's lead. Island Press. Washington, EU. Pp. 256.

Tylianakis, JM; T TscharntKe \& OT LeWIs. 2007. Habitat modification alters the structure of tropical hostparasitoid food webs. Nature, 445:202-205.

Verdú, M \& A Valiente-Banuet. 2008. The nested assembly of plant facilitation networks prevents species extinctions. Am Nat, 172:751-760.

WAJNBERG, E; C BERNSTEIN \& J vAN AlPHEN. 2008. Behavioral ecology of insect parasitoids from theoretical approaches to field applications. Wiley-Blackwell Publishing. Oxford, UK. Pp. 445. 